\title{
HEAT DIFFUSION ANALYSIS IN SINGLE-CRYSTAL AND POLY-CRYSTAL MATERIAL SURFACES BY INFRARED THERMOGRAPHY
}

\author{
M. R. Tejaswini ${ }^{1}$, Prof. K. Balasubramaniam ${ }^{1}$, Prof. C. V. Krishnamurthy ${ }^{2}$
}

\author{
${ }^{1}$ CNDE, MDS, Mechanical Dept., Indian Institute of Technology Madras, Chennai- 36 \\ ${ }^{2}$ Dept. of Physics, Indian Institute of Technology Madras, Chennai- 36
}

\begin{abstract}
Thermal diffusivity is one of the most important parameters when heat transfer phenomena are involved [1]. The complete characterization of time-varying thermal phenomena requires the knowledge of thermal diffusivity [2]. Study of thermal property of microstructures also requires thermal study of grains of the material (polycrystalline) involved. We report in-plane thermal diffusivity of single-crystal and poly-crystal material (lateral dimensions >>thickness) with pulsed laser spot heating. The thermographic technique used is based on instantaneous heating of the front surface by a heating source and detection of the spatial distribution of the temperature of the rear surface of a sample [3]. Heating source is assumed to be Gaussian for analysis purpose and for simulations. The samples used are single crystal silicon, steel and polysilicon, each having thickness of a few hundreds of microns. The Gaussian source is a 3ns Q-switched Nd-YAG laser, heat diffusion is monitored by a LWIR infrared camera with a frame rate of about $1 \mathrm{kHz}$. Polysilicon used contains grains of the order of $1 \mathrm{~cm}$ which are visible by eye. Thermal diffusivity in a grain of a material can be found in the same way mentioned above. Measurement along different lateral direction shows anisotropy in the value of thermal diffusivity. The experiment has been repeated for few such grains in the material. The experiments will be backed by simulation.
\end{abstract}

Keywords: Heat diffusion, Thermal diffusivity, Grain, Anisotropy, Thermography.

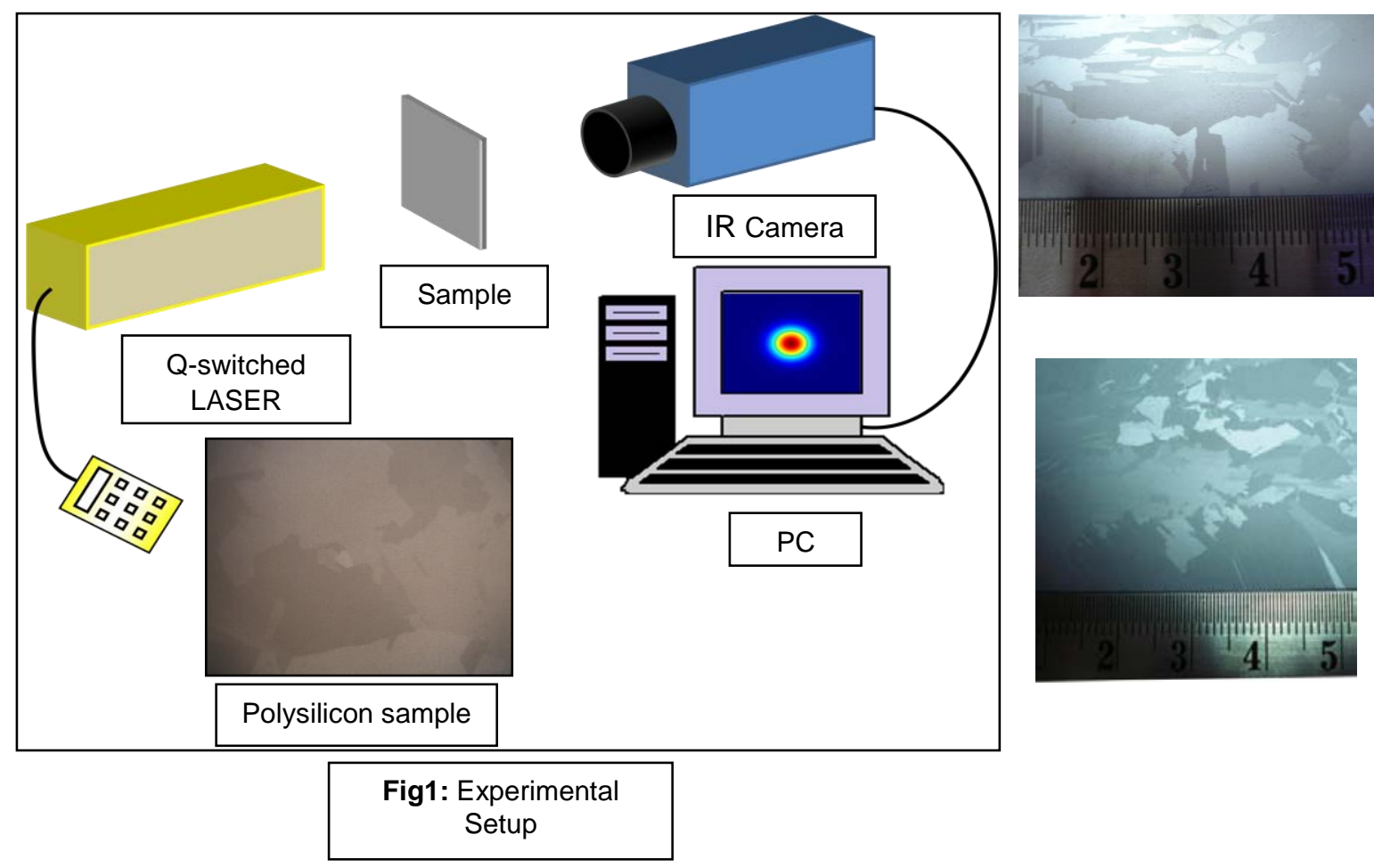

\section{Experimental result}

Figure 1 shows the experimental setup used. A Quantel BRIO make, Q switched Nd-YAG Laser of $532 \mathrm{~nm}, 3 \mathrm{~ns}$ pulse width is used to deposit heat on a grain. An IR camera (Cedip, JADE) with sufficiently high frame rate (around $1 \mathrm{kHz}$ ) to capture the diffusion process is used on the rear end to monitor. Camera is connected to a system that has software to control the camera (Cirrus software) and receive data (Altair) from it. Result is shown for single crystal silicon and polysilicon (single grain). Figure 4 and 5 show the heat diffusion and line profiles from which thermal diffusivity value is calculated.

Heat diffusion will be modeled in 3D using the general heat transfer physics of COMSOL 3.5a. For a single crystal silicon heat diffusion model, the following are the properties used : $k=130 \mathrm{~W} / \mathrm{m} . \mathrm{K}$, density, $\rho=2330 \mathrm{~kg} / \mathrm{m}^{3}$, specific heat, $c_{p}=697.4248 \mathrm{~J} / \mathrm{kg} . \mathrm{K}$. Figure 2 gives the back surface temperature profile and line profiles at consecutive frames.. Table 1 gives the values of thermal diffusivity in different direction for different grains. 


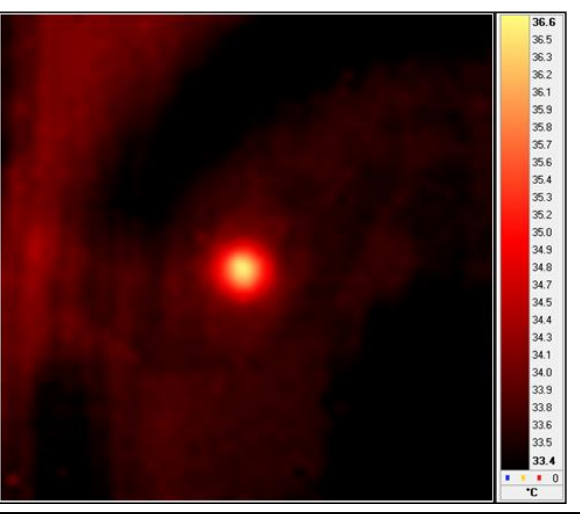

Fig4: Heat diffusion in a grain at the back surface of the polysilicon sample, immediately after the laser pulse.
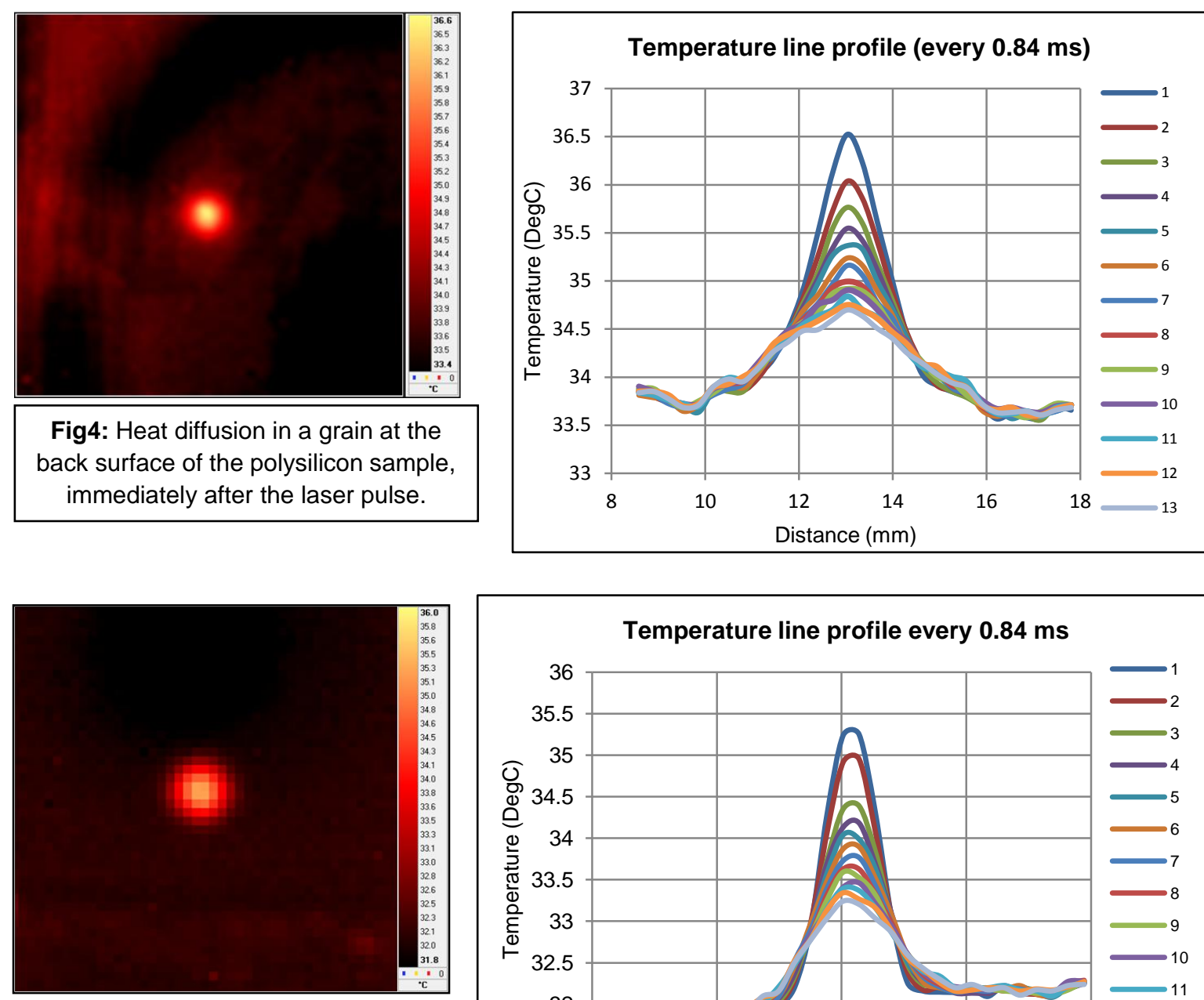

Fig4: Heat diffusion at the back surface of the Silicon sample, immediately after the laser pulse.

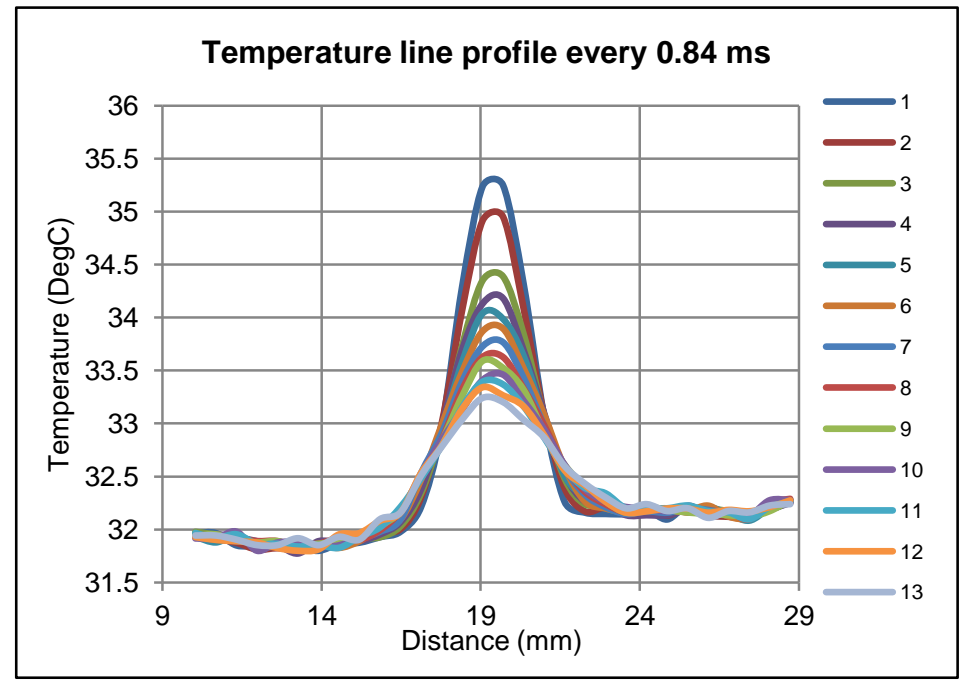

Summary and inference

A non contact method has been used, to find the in-plane thermal diffusivity of thin materials also having small lateral dimension (about $10 \mathrm{~mm}$, lateral dimension >> thickness). So this method can be used to find thermal diffusivity of small materials, or study local thermal diffusion in case of large materials. The materials used are typical, in the sense that steel covers the mechanical industry and silicon covers the electronic industry. The method used to determine thermal diffusivity proves to be reliable, giving reasonably good agreement with literature values. The percentage deviation from actual value for Silicon is $3.83 \%$ and steel is $4.44 \%$. From the thermal diffusivity in different direction, an estimate of thermal diffusion anisotropy in polysilicon can be made. Further work has to be done on these lines.

\section{REFERENCES}

1. H. S. Carslaw and J. C. Jaeger, Conduction of Heat in Solids, 2nd ed.(Clarendon, Oxford, 1959), No. 29.

2. A. Salazar , 'On thermal diffusivity', Eur. J. Phys. 24 (2003) 351-358.

3. F. Cernuschi, A. Russo, L. Lorenzoni and A. Figari, 'In-plane thermal diffusivity evaluation by infrared thermography', REVIEW OF SCIENTIFIC INSTRUMENTS, 72(10), 3988- 3995, 2001. 